\title{
NORMAL SUBGROUPS OF THE GENERAL LINEAR GROUPS OVER VON NEUMANN REGULAR RINGS
}

\author{
L. N. VASERSTEIN ${ }^{1}$
}

\begin{abstract}
Let $A$ be a von Neumann regular ring or, more generally, let $A$ be an associative ring with 1 whose reduction modulo its Jacobson radical is von Neumann regular. We obtain a complete description of all subgroups of $\mathrm{GL}_{n} A, n \geq 3$, which are normalized by elementary matrices.
\end{abstract}

1. Introduction. For any associative ring $A$ with 1 and any natural number $n$, let $\mathrm{GL}_{n} A$ be the group of invertible $n$ by $n$ matrices over $A$ and $E_{n} A$ the subgroup generated by all elementary matrices $x^{i, j}$, where $1 \leq i \neq j \leq n$ and $x \in A$.

In this paper we describe all subgroups of $\mathrm{GL}_{n} A$ normalized by $E_{n} A$ for any von Neumann regular $A$, provided $n \geq 3$. Our description is standard (see Bass $[\mathbf{1}]$ and Vaserstein $[14,16])$ : a subgroup $H$ of $\mathrm{GL}_{n} A$ is normalized by $E_{n} A$ if and only if $H$ is of level $B$ for an ideal $B$ of $A$, i.e. $E_{n}(A, B) \subset H \subset G_{n}(A, B)$. Here $G_{n}(A, B)$ is the inverse image of the center of $\mathrm{GL}_{n}(A / B$ ) (when $n \geq 2$, this center consists of scalar invertible matrices over the center of the ring $A / B$ ) under the canonical homomorphism $\mathrm{GL}_{n} A \rightarrow \mathrm{GL}_{n}(A / B)$ and $E_{n}(A, B)$ is the normal subgroup of $E_{n} A$ generated by all elementary matrices in $G_{n}(A, B)$ (when $n \geq 3$, the group $E_{n}(A, B)$ is generated by matrices of the form $(-y)^{j, i} x^{i, j} y^{j, i}$ with $x \in B, y \in A, 1 \leq i \neq j \leq n$, see $[\mathbf{1 4}])$.

Recall that a ring $A$ is called von Neumann regular (see von Neumann [13], Goodearl [7]) if for any $z$ in $A$ there is $x$ in $A$ such that $z x z=z$. Then every factor ring and every ideal of $A$ is also von Neumann regular.

In fact, to be more general, we assume that $A / \operatorname{rad}(A)$ (rather than $A$ ) is von Neumann regular, where rad means the Jacobson radical. For example, this assumption holds for any Artinian ring $A$ or for any commutative semilocal ring $A$.

THEOREM 1. Assume that $A / \operatorname{rad}(A)$ is von Neumann regular and $n \geq 2$. Then for any ideal $B$ of $A$ :

(a) $E_{n}(A, B)$ contains all matrices of the form $1_{n}+v u$, where $v$ is an $n$-column over $A, u$ is an $n$-row over $B$, and $u v=0$; in particular, $E_{n}(A, B)$ is normal in $\mathrm{GL}_{n} A$;

(b) $E_{n}(A, B) \supset\left[E_{n} A, G_{n}(A, B)\right]$; in particular, every subgroup of $\mathrm{GL}_{n} A$ of level $B$ is normalized by $E_{n} A$;

(c) if $n \geq 3$, we have $E_{n}(A, B)=\left[E_{n} A, E_{n} B\right]=\left[\mathrm{GL}_{n} A, E_{n}(A, B)\right]=\left[E_{n} A, H\right]$ for any subgroup $H$ of level $B$, where $E_{n} B$ is the subgroup of $G_{n}(A, B)$ generated by elementary matrices;

Received by the editors February 13, 1985. Presented to the Society, April 21, 1985 at the 819th meeting.

1980 Mathematics Subject Classification. Primary 16A54, 18F25, 16A30.

${ }^{1}$ Supported by National Science and Guggenheim Foundations. 
(d) if $A$ is von Neumann regular, we have $E_{n} B=E_{n}(A, B)$; if moreover, $n \geq 3$, we have $E_{n} B=\left[E_{n} B, E_{n} B\right]$.

THEOREM 2. Assume that $A / \operatorname{rad}(A)$ is von Neumann regular and $n \geq 3$. Then every subgroup $H$ of $\mathrm{GL}_{n} A$ normalized by $E_{n} A$ is of level $B$ for some ideal $B$ of $A$, i.e. $E_{n}(A, B) \subset H \subset G_{n}(A, B)$.

Note that a subgroup $H$ of $\mathrm{GL}_{n} A, n \geq 2$, cannot be of level $B$ and of level $B^{\prime}$ for two distinct ideals $B$ and $B^{\prime}$ of $A$. So the level $B$ in Theorem 2 is unqiue.

Theorems 1 and 2 were proved by Dickson [2] when $A$ is a field (the condition $n \geq 3$ in this case can be replaced by the condition card $(A) \geq 4)$, by Dieudonné $[3]$ when $A$ is a division ring, by Klingenberg $[10]$ when $A$ is a commutative local ring, by Bass [1] when $A$ satisfies the stable range condition $\operatorname{sr}(A) \leq n-1$, by Vaserstein [14] when central localizations of $A$ satisfy this stable range condition (for example, when $A$ is finite as module over its center) and $n \geq 3$, and by Vaserstein [16] when $A$ is a Banach algebra. Theorem 2 is claimed by Golubchik $[\mathbf{5}, \mathbf{6}]$ under the additional condition that $A / M$ is an Ore ring for every maximal ideal $M$ of $A$.

Note that von Neumann regular rings $A$ satisfying $\operatorname{sr}(A) \leq 1$ are known as unit regular rings, see $[\mathbf{7}, \mathbf{8}, \mathbf{9}, \mathbf{1 1}, \mathbf{1 2}, \mathbf{1 5}]$.

2. Proof of Theorem 1(a). We write

$$
v=\left(v_{i}\right)=\left(\begin{array}{c}
v^{\prime} \\
v_{n}
\end{array}\right) \quad \text { and } \quad u=\left(u_{j}\right)=\left(u^{\prime}, u_{n}\right)
$$

with $v_{i}$ in $A$ and $u_{j}$ in $B$.

Case 1. $1+v_{n} u_{n} \in \mathrm{GL}_{1} B$. We set $d:=1+v_{n} u_{n}, d^{\prime}:=1+u_{n} v_{n}=$ $1-u^{\prime} v^{\prime} \in \mathrm{GL}_{1} B$ (see $\left.[\mathbf{1 7}, \S 2]\right)$ and $a=1_{n-1}+v^{\prime} u^{\prime}-v^{\prime} u_{n} d^{-1} v_{n} u^{\prime}=1_{n-1}+$ $v^{\prime}\left(1-u_{n} d^{-1} v_{n}\right) u^{\prime}=1_{n-1}+v^{\prime} d^{\prime-1} u^{\prime}$. Then

$$
\begin{aligned}
1_{n}+v u & =\left(\begin{array}{cc}
1_{n-1}+v^{\prime} u^{\prime} & v^{\prime} u_{n} \\
v_{n} y^{\prime} & d
\end{array}\right) \\
& =\left(\begin{array}{cc}
1_{n-1} & v^{\prime} u_{n} d-1 \\
0 & 1
\end{array}\right)\left(\begin{array}{ll}
a & 0 \\
0 & d
\end{array}\right)\left(\begin{array}{cc}
1_{n-1} & 0 \\
d^{-1} v_{n} u^{\prime} & 1
\end{array}\right) \\
& \in E_{n} B\left(\begin{array}{ll}
a & 0 \\
0 & d
\end{array}\right) E_{n} B .
\end{aligned}
$$

We have to prove that $\left(\begin{array}{ll}a & 0 \\ 0 & d\end{array}\right) \in E_{n}(A, B)$.

Since $1+u^{\prime} v^{\prime} d^{\prime-1}=d^{\prime-1}$, we have

$$
\begin{aligned}
\left(\begin{array}{ll}
a & 0 \\
0 & 1
\end{array}\right)= & \left(\begin{array}{cc}
1_{n-1} & 0 \\
u^{\prime} a^{-1} & 1
\end{array}\right)\left(\begin{array}{cc}
1_{n-1} & -v^{\prime} d^{\prime-1} \\
0 & 1
\end{array}\right)\left(\begin{array}{cc}
1_{n} & 0 \\
-u^{\prime} & 1
\end{array}\right) \\
& \cdot\left(\begin{array}{cc}
1_{n-1} & v^{\prime} \\
0 & 1
\end{array}\right)\left(\begin{array}{cc}
1_{n-1} & 0 \\
0 & d^{\prime-1}
\end{array}\right) \\
\in & E_{n}(A, B)\left(\begin{array}{cc}
1_{n-1} & 0 \\
0 & d^{\prime-1}
\end{array}\right) .
\end{aligned}
$$


By $[17, \S 2]$,

$$
\left(\begin{array}{cc}
1_{n-1} & 0 \\
0 & d^{\prime-1} d
\end{array}\right) \in E_{n}(A, B) .
$$

So $1_{n}+v u \in E_{n}(A, B)$ in Case 1 .

Case 2. $v_{i} \in \operatorname{rad}(A)$ for some $i$ with $1 \leq i \leq n$. Since $E_{n}(A, B)$ is normalized by all permutation matrices, we can assume that $i=n$. Then $1+v_{n} u_{n} \in \mathrm{GL}_{1} B$, so we are reduced to Case 1.

General case. We now use the condition that $A / \operatorname{rad}(A)$ is von Neumann regular, hence there is an $x$ in $A$ such that $v_{n} x v_{n}-v_{n} \in \operatorname{rad}(A)$. Then $1+v_{n}\left(1-x v_{n}\right) u_{n} \in$ $\mathrm{GL}_{1} B$, hence $g:=1_{n}+v\left(1-x v_{n}\right) u \in \mathrm{GL}_{n} B$ by Case 1.

Also we have

$$
\begin{aligned}
& \left(-v_{n-1} x\right)^{n-1, n}\left(1_{n}+v x v_{n} u\right)\left(v_{n-1} x\right)^{n-1, n} \\
& \quad=1_{n}+\left(\left(-v_{n-1} x\right)^{n-1, n} v x v_{n-1}\right)\left(u\left(v_{n-1} x\right)^{n-1, n}\right)
\end{aligned}
$$

and

$$
\left(\left(-v_{n-1} x\right)^{n-1, n} v x v_{n}\right)_{n-1}=v_{n-1}\left(1-x v_{n}\right) x v_{n}=v_{n-1} x\left(v_{n}-v_{n} x v_{n}\right) \in \operatorname{rad}(A),
$$

hence $h:=1_{n}+v x v_{n} u \in E_{n}(A, B)$ by Case 2 with $i=n-1$.

Therefore $1_{n}+v u=g h \in E_{n}(A, B)$.

3. Proof of Theorem 1(b). It suffices to show that $\left[y^{i, j}, g\right]:=y^{i, j} g(-y)^{i, j} g^{-1}$ $\in E_{n}(A, B)$ for any elementary $y^{i, j}$ in $E_{n} A$ and any $g$ in $G_{n}(A, B)$. Since $E_{n}(A, B)$ is norr alized by all permutation matrices, we can assume that $(i, j)=(1, n)$.

Then $\left[y^{i, j}, g\right]=y^{1, n}\left(1_{n}-v y w\right)$, where $v=\left(\begin{array}{l}v^{\prime} \\ v_{n}\end{array}\right)$ is the first column of $g$ and $w=\left(w^{\prime}, w_{n}\right)$ is the last row of $g^{-1}$, so $w v=0$.

As in the end of the previous section, we find $x$ in $A$ such that $v_{n} x v_{n}-v_{n} \in$ $\operatorname{rad}(A)$, and we have $h:=1_{n}-v x v_{n} w \in E_{n}(A, B)$, hence

$$
\left[\left(x v_{n}\right)^{1, n}, g\right]=\left(x v_{n}\right)^{1, n}\left(1_{n}-v x v_{n} w\right) \in E_{n}(A, B),
$$

i.e. $\left(x v_{n}\right)^{1, n}$ and $g$ commute modulo $E_{n}(A, B)$.

To complete our proof, it suffices to show that $\left(1-x v_{n}\right)^{1, n}$ also commutes with $g$ modulo $E_{n}(A, B)$. We set $u:=-\left(1-x v_{n}\right) w=\left(u^{\prime}, u_{n}\right)$. Then

$$
\left[\left(1-x v_{n}\right)^{1, n}, g\right]=\left(1-x v_{n}\right)^{1, n}\left(1_{n}+v u\right),
$$

with $v_{n} u_{n}=v_{n}\left(1-x v_{n}\right) w_{n} \in \operatorname{rad}(B)$, hence $d:=1+v_{n} u_{n} \in \mathrm{GL}_{1} B$. Also $v_{i} \in B$ for $i \geq 2, u_{j} \in B$ for $j \leq n-1$ and $v_{1} u_{n}+1 \in B$.

We set $d^{\prime}:=1+u_{n} v_{n}=1-u^{\prime} v^{\prime} \in \mathrm{GL}_{1} B$ and $a:=1_{n-1}+v^{\prime} u^{\prime}-v^{\prime} u_{n} d^{-1} v_{n} u^{\prime}=$ $1_{n-1}+v^{\prime} d^{\prime-1} u^{\prime}$. Then

$$
\begin{aligned}
\left(1-x v_{n}\right)^{1, n}\left(1_{n}+v u\right) & =\left(1-x v_{n}\right)^{1, n}\left(\begin{array}{cc}
1_{n-1} & v^{\prime} u_{n} d^{-1} \\
0 & 1
\end{array}\right)\left(\begin{array}{ll}
a & 0 \\
0 & d
\end{array}\right)\left(\begin{array}{cc}
1_{n-1} & 0 \\
d^{-1} v_{n} u^{\prime} & 1
\end{array}\right) \\
& \in E_{n} B\left(\begin{array}{ll}
a & 0 \\
0 & d
\end{array}\right) E_{n} B .
\end{aligned}
$$

Now, as in the previous section (see Case 1 there), we see that $\left(\begin{array}{ll}a & 0 \\ 0 & d\end{array}\right) \in E_{n}(A, B)$. (Note that $u^{\prime}$ is an $(n-1)$-row over $B$.) 
4. Proof of Theorem 1(c). In the view of Theorem 1(a), (b), we have only the inclusion $E_{n} B \subset\left[E_{n} A, E_{n} B\right]$ to prove. But we have it for any ring $A$ with 1 and any $n \geq 3$ by the formula $x^{i, j}=\left[1^{i, k}, x^{k, j}\right]$, where $1 \leq i \neq j \neq k \neq i \leq n$ and $x \in B$.

5. Proof of Theorem $\mathbf{1}(\mathbf{d})$. We want to prove first that $E_{n}(A, B)=E_{n} B$, i.e. $E_{n} B$ is normalized by every elementary matrix $y^{i, j}$ in $\mathrm{GL}_{n} A$. Since $E_{n} B$ is normalized by all permutation matrices, we can assume that $(i, j)=(1,2)$. It suffices to prove that $h:=(-y)^{1,2} g y^{1,2} \in E_{n} B$ for every elementary matrix $g$ in $E_{n} B$. This is trivial (and true for an arbitrary ring $A$ ) unless $g=z^{2,1}$ where $z \in B$. In this case we can assume that $n=2$.

Since $A$ is von Neumann regular, $z=z x z$ for some $x$ in $A$. We have

$$
h=(-y)^{1,2} z^{2,1} y^{1,2}=(-x z y)^{1,2}(x z y-y)^{1,2} z^{2,1}(y-x z y)^{1,2}(x z y)^{1,2} .
$$

But $(x z y)^{1,2} \in E_{2} B$ and

$$
\begin{aligned}
(x z y-y)^{1,2} z^{2,1}(y-x z y)^{1,2} & =\left(\begin{array}{cc}
1+(x z-1) y z & 0 \\
z & 1
\end{array}\right) \\
& =((x z-1) y z x)^{1,2} z^{2,1}((1-x z) y z)^{1,2} \in E_{2} B .
\end{aligned}
$$

When $n \geq 3$, for any elementary $z^{i, j}$ in $E_{n} B$ we have $z^{i, j}=\left[(z x)^{i, k}, z^{k, j}\right]$, where $k \neq i, j$ and $z=z x z$ with $x$ in $A$.

6. Proof of Theorem 2. Let $H$ be a subgroup of $\mathrm{GL}_{n} A$ normalized by $E_{n} A$, where $n \geq 3$. The condition that $A / \operatorname{rad}(A)$ is von Neumann regular will not be used in Cases 1-5 of Lemma 3 below or Lemma 4 .

LEMMA 3. If $H$ is not central, then $H$ contains an elementary matrix $\neq 1_{n}$.

Proof. Case 1. $H \ni g=\left(g_{i, j}\right)$ such that $g_{n, 1}=0$ and $g$ does not commute with some $1^{k, 1} \in E_{n} A$. Then $H$ contains an elementary matrix $\neq 1_{n}$ by Vaserstein $[\mathbf{1 4}]$.

Case 2. $H \ni h=\left(h_{i, j}\right)$ such that $h_{n, 2} \neq 0$ and $h_{n, 1}+h_{n, 2} y=0$ for some $y$ in $A$. Then $H \ni(-y)^{2,1} h x^{2,1}=: g=\left(g_{i, j}\right)$ and $g_{n, 1}=h_{n, 1}+h_{n, 2} y=0, g_{n, 2}=h_{n, 2} \neq 0$, so $\left[g, 1^{2,1}\right] \neq 1_{n}$. Thus, we are reduced to Case 1 .

Case 3. $H$ contains a noncentral $g=\left(g_{i, j}\right)$ with $g_{n, 1}=0$. If $g$ does not commute with some $1^{k, 1} \in E_{n} A$, we are done by Case 1 . Otherwise, $g$ is a scalar matrix: $g_{i, j}=0=g_{i, i}-g_{j, j}$ for all $i \neq j$. Since $g$ does not belong to the center of $\mathrm{GL}_{n} A$, there is $y$ in $A$ such that $y g_{1,1} \neq g_{1,1} y$. Then $\left[g, y^{1,2}\right]=\left(g_{1,1} y-y g_{2,2}\right)^{1,2} \neq 1_{n}$ is an elementary matrix in $H$.

Case 4. $H$ contains a noncentral $h=\left(h_{i, j}\right)$ with $h_{2,2} \in \mathrm{GL}_{1} A$. If $\left(h^{-1}\right)_{n, 1}=0$, we are done by Case 3 with $g=h^{-1}$. Otherwise, $H \ni(-1)^{1,2} 1^{1,2} h=\left(g_{i, j}\right)$ with $\left(g_{n, 1}, g_{n, 2}\right)=\left(h^{-1}\right)_{n, 1}\left(h_{2,1}, h_{2,2}\right)$, so we are reduced to Case 2 .

Case 5. $H$ contains a noncentral $h=\left(h_{i, j}\right)$ with $h_{n, 2}=0$. Since $f:=\left(\begin{array}{cc}0 & 1 \\ -1 & 0\end{array}\right)=$ $\left(\begin{array}{ll}1 & 1 \\ 0 & 1\end{array}\right)\left(\begin{array}{cc}1 & 0 \\ -1 & 1\end{array}\right)\left(\begin{array}{ll}1 & 1 \\ 0 & 1\end{array}\right) \in E_{2} A$, we have $f^{\prime}:=\left(\begin{array}{cc}f & 0 \\ 0 & 11_{n-2}\end{array}\right) \in E_{n} A$ and $g:=f^{\prime} h f^{\prime-1} \in H$. Since $g_{n, 1}=h_{n, 2}=0$, we are reduced to Case 3 .

General case. We pick a noncentral $h=\left(h_{i, j}\right)$ in $H$ and find $x$ in $A$ such that $z:=h_{n, 2} x h_{n, 2}-h_{n, 2} \in \operatorname{rad}(A)$. We set $p:=1-h_{n, 2} x$. If $p h_{n, 1}=0$, i.e. $h_{n, 1}-h_{n, 2} x h_{n, 1}=0$, then we are done by Case 5 or Case 2 . Otherwise, the matrix 
$g=\left(g_{i, j}\right):=h^{-1} p^{1, n} h(-p)^{1, n} \in H$ is not central and $g_{2,2}=1+\left(h^{-1}\right)_{2,1} p h_{n, 2}=$ $1-\left(h^{-1}\right)_{2,1} z \in \mathrm{GL}_{1} A$, so we are reduced to Case 4 .

LEMMA 4. If $H \ni x^{i, j}$, where $x \in A, 1 \leq i \neq j \leq n$, then $H \supset E_{n}(A, B)$, where $B$ is the (two-sided) ideal of $A$ generated by $x$.

ProOF. It follows easily from the identities $y^{i, j} z^{i, j}=(y+z)^{i, j}$ and $\left[y^{i, j}, z^{j, k}\right]=$ $(y z)^{i, k}$, where $1 \leq i \leq j \neq k \neq i \leq n$ and $y, z$ are in $A$ (we use here that $n \geq 3$; no conditions on $A$ are needed).

Now we can conclude our proof of Theorem 2. By Lemma 4, there is an ideal $B$ of $A$ such that $E_{n}(A, B)$ contains all elementary matrices in $H$. Consider the image $H^{\prime}$ of $H$ in $\mathrm{GL}_{n}(A / B)$. Since the $\operatorname{ring}(A / B) / \operatorname{rad}(A / B)$ is a factor ring of $A / \operatorname{rad}(A)$, it is also von Neumann regular. Since $H^{\prime}$ is normalized by $E_{n}(A / B)$ which is the image of $E_{n} A$, Lemma 3 applied to $H^{\prime}$ gives that either $H^{\prime}$ is central or $H^{\prime}$ contains an elementary matrix $\left(x^{\prime}\right)^{i, j}$, where $0 \neq x^{\prime} \in A / B$ and $1 \leq i \neq j \leq n$. In the latter case, $H \ni x^{i, j} g$, where $0 \neq x \in A, x^{\prime}=x+B$, and $g \in \mathrm{GL}_{n} B$. We pick an integer $k \neq i, j$ in the interval $1 \leq k \leq n$. Then $H \ni\left[x^{i, j} g, 1^{j, k}\right]=x^{i, k} 1^{j, k} x^{i, j}\left[(-1)^{j, k}, g\right](-x)^{i, j}(-1)^{j, k} \in x^{i, k} E_{n}(A, B) \subset x^{i, k} H$ by Theorem 1(b). Therefore $H \ni x^{i, k}$ which contradicts our choice of $B$.

Thus, $H^{\prime}$ is central in $\mathrm{GL}_{n}(A / B)$, i.e. $H \subset G_{n}(A, B)$.

REMARK. From the proof of Theorem 1(a) (see $\S 2$ above), it is clear that the group $E_{n}(A, B)$ is generated by matrices of the form $(-y)^{j, i} x^{i, j} y^{j, i}$ with $x$ in $B$ and $y$ in $A$, provided $n \geq 2$ and $A / \operatorname{rad}(A)$ is von Neumann regular. If $n \geq 3$, no restrictions on $A$ are needed.

\section{REFERENCES}

1. H. Bass, K-theory and stable algebra, Publ. Math. Inst. Hautes Etudes Sci. 22 (1964), 5-60.

2. L. E. Dickson, Theory of linear groups in an arbitrary field, Trans. Amer. Math. Soc. 2 (1901), 363-394.

3. J. Dieudonné, Le géométrie des groupes classiques, 3rd ed., Springer, New York and Berlin, 1971.

4. G. Ehrlich, Units and one-sided units in regular rings, Trans. Amer. Math. Soc. 216 (1976), 81-90.

5. I. Z. Golubchik, Normal subgroups of linear groups over rings, Vestnik Moskov Univ. Ser. I Mat. Mekh. 1978, no. 6, 79. (Russian)

6. I. Z. Golubchik and A. V. Mikhalev, Epimorphisms of projective groups over associative rings, Algebra, Moskov Univ., 1982, pp. 34-45.

7. K. R. Goodearl, Von Neumann regular rings, Pitman, London, San Francisco and Melbourne, 1979.

8. M. Henriksen, On a class of regular rings that are elementary divisor rings, Arch. Math. 24 (1973), 133-141.

9. R. Hartwig and J. Luh, A note on the group structure of unit regular ring elements, Pacific J. Math. 71 (1977), 449-461.

10. W. Klingenberg, Lineare Gruppen über lokalen Ringen, Amer. J. Math. 83 (1961), 137-153.

11. P. Menal and J. Moncasi, On regular rings with stable range 2, J. Pure Appl. Algebra 24 (1982), 25-40.

12. __ $K_{1}$ of von Neumann regular rings, J. Pure Appl. Algebra 33 (1984), 295-312.

13. J. von Neumann, On regular rings, Proc. Nat. Acad. Sci. U.S.A. 22 (1936), 707-713.

14. L. N. Vaserstein, On normal subgroups of $\mathrm{GL}_{n}$ over a ring, Lecture Notes in Math., vol. 854, Springer-Verlag, Berlin and New York, 1981, pp. 456-465.

15. __ Bass's first stable range condition, J. Pure Appl. Algebra 34 (1984), 319-330. 
16. __ Normal subgroups of the general linear groups over Banach algebras, preprint, Institute for Advanced Study, January 1985; J. Pure Appl. Algebra (to appear).

17. _ $K_{1}$-theory and the congruence subgroup problem, Mat. Zametki 5 (1969), 233$244=$ Math. Notes 5, 141-148.

Department of Mathematics, Pennsylvania State University, University PARK, PENNSYLVANIA 16802 (Current address)

The Institute for AdVanced Study, Princeton, New Jersey 08540 